\title{
Piperazine ferulate prevents high-glucose-induced filtration barrier injury of glomerular endothelial cells
}

\author{
YONG-YU YANG ${ }^{1,2}$, ZHUO CHEN $^{3}$, XI-DING YANG ${ }^{1}$, RONG-RONG DENG ${ }^{4}$, \\ LING-XING SHI ${ }^{5}$, LIANG-YUAN YAO ${ }^{6}$ and DA-XIONG XIANG ${ }^{1,2}$ \\ ${ }^{1}$ Department of Pharmacy, ${ }^{2}$ Hunan Provincial Engineering Research Central of Translational Medical and Innovative Drug, \\ The Second Xiangya Hospital of Central South University, Changsha, Hunan 410011; \\ ${ }^{3}$ Department of Geriatrics, The Third Xiangya Hospital of Central South University, Changsha, Hunan 410013; \\ ${ }^{4}$ School of Traditional Chinese Medicine, Guangdong Pharmaceutical University, Guangzhou, Guangdong 510006; \\ ${ }^{5}$ Department of Pharmacology, Changsha Medical University, Changsha, Hunan 410219; \\ ${ }^{6}$ Hunan Qianjin Xiangjiang Pharmaceutical Industry Co., Ltd., Zhuzhou, Hunan 412000, P.R. China
}

Received February 19, 2021; Accepted July 19, 2021

DOI: 10.3892/etm.2021.10607

\begin{abstract}
Filtration barrier injury induced by high glucose (HG) levels leads to the development of diabetic nephropathy. The endothelial glycocalyx plays a critical role in glomerular barrier function. In the present study, the effects of piperazine ferulate $(\mathrm{PF})$ on $\mathrm{HG}$-induced filtration barrier injury of glomerular endothelial cells (GEnCs) were investigated and the underlying mechanism was assessed. Immunofluorescence was used to observe the distribution of the glycocalyx as well as the expression levels of syndecan-1 and Zonula occludens-1 (ZO-1). Endothelial permeability assays were performed to assess the effects of PF on the integrity of the filtration barrier. Protein and mRNA expression levels were measured by western blotting and reverse transcription-quantitative PCR analyses, respectively. In vitro experiments revealed that adenosine monophosphate-activated protein kinase (AMPK) mediated HG-induced glycocalyx degradation and endothelial barrier injury. PF inhibited the HG-induced endothelial barrier injury and restored the expression levels of heparanase-1 (Hpa-1), ZO-1 and occludin-1 by AMPK. In vivo assays demonstrated that PF reduced the expression levels of Hpa-1, increased the expression levels of ZO-1 and attenuated glycocalyx degradation in the glomerulus. These data suggested that PF attenuated HG-induced filtration barrier injury of GEnC by regulating AMPK expression.
\end{abstract}

Correspondence to: Professor Da-Xiong Xiang, Department of Pharmacy, The Second Xiangya Hospital of Central South University, 139 Renmin Middle Road, Changsha, Hunan 410011, P.R. China E-mail: xiangdaxiong@csu.edu.cn

Key words: filtration barrier, piperazine ferulate, diabetic nephropathy, adenosine monophosphate-activated protein kinase, glycocalyx, tight junction

\section{Introduction}

Diabetic nephropathy (DN) is associated with damage to the glomerular filtration barrier (GFB), which consists of the three following layers: The endothelial layer, the glomerular basement membrane and the podocytes $(1,2)$. GFB is responsible for filtering water and small molecules from circulating plasma. Accumulating evidence has demonstrated that glomerular endothelial cells (GEnCs) act as the first barrier to prevent macromolecules from passing the $\operatorname{GFB}(2,3)$. Glycocalyx is the main component of the endothelial surface layer, which constitutes a molecular size- and charge-specific selective barrier together with the fenestrations $(4,5)$. The degradation of the glycocalyx and the decreased number of fenestrations are the most important characteristics of filtration barrier injury to GEnCs during DN (6). Since glycocalyx dysfunction exerts an important role in the development of DN, the prevention of glycocalyx degradation may be an alternative approach to treat DN.

Adenosine monophosphate-activated protein kinase (AMPK) is a ubiquitous heterotrimeric protein composed of a catalytic subunit $(\alpha)$ and two regulatory subunits $(\beta$ and $\gamma)(7)$. A previous study demonstrated that AMPK is an important molecule that regulates the progression of DN (8). Renal hypertrophy (9) and podocyte apoptosis (10) in diabetes are regulated by AMPK, whereas metformin, an activator of AMPK, has been demonstrated to decrease albuminuria in diabetic rats as well as in patients with type 2 diabetes mellitus $(11,12)$. Furthermore, AMPK has been shown to mediate low shear stress-induced glycocalyx impairment (13). This evidence indicates that AMPK may be a possible drug target to prevent glomerular filtration injury.

Piperazine ferulate (Piperazine 3-methoxy-4-hydroxycinnamate; PF; Fig. 1A) is a derivative of ferulic acid that exhibits anti-hypertensive effects (14), reduces IgA-mediated nephropathy (15) and prevents the development of DN (16). A systematic meta-analysis indicated that PF combined with irbesartan could improve the efficiency of treatment 
of DN (17). Our previous study also demonstrated that PF retarded the progression of DN (16) and attenuated the high glucose (HG)-induced mesangial cell injury (18). However, it remains unclear whether the mechanism of PF against $\mathrm{DN}$ is associated with the regulation of GEnC filtration barrier injury. In the present study, the effects of PF on the HG-induced filtration barrier injury of GEnCs were investigated. Moreover, the potential mechanism of its action was assessed.

\section{Materials and methods}

Materials. PF was provided by Hunan QianJinXiangJiang Pharmaceutical Industry Co., Ltd. Fluorescein isothiocyanate (FITC)-dextran and FITC conjugated wheat germ agglutinin (FITC-WGA) were obtained from Sigma-Aldrich (Merck KGaA). Rabbit polyclonal anti-occludin-1 (cat. no. DF7504) and anti-heparanase (Hpa-1) antibodies (cat. no. DF12411) were purchased from Affinity Biosciences. Rabbit polyclonal anti-AMPK (cat.no.2532) and anti-phosphorylated (p)-AMPK $\alpha$ (Thr172) antibodies (cat. no. 2535) were obtained from Cell Signaling Technology, Inc. Rabbit polyclonal anti-Zonula occludens-1 (ZO-1; cat. no. PB9234) and mouse monoclonal anti- $\beta$-actin antibody (cat. no. BM0627), as well as the syndecan-1 (Sdc-1) ELISA kits (cat. no. EK1339 and EK1554) were purchased from Boster Biological Technology, Ltd. $\mathrm{SYBR}^{\circledR}$ Premix Ex Taq $^{\mathrm{TM}}$ and PrimeScript reverse transcription reagent kit were obtained from Takara Bio, Inc. TRIzol ${ }^{\circledR}$ was obtained from Thermo Fisher Scientific, Inc. RIPA lysis buffer, 5-aminoimidizole-4-carboxamide riboside (AICAR), the bicinchoninic acid (BCA) assay kit and a BeyoECL Plus kit were obtained from Beyotime Institute of Biotechnology. Glucose and mannitol were purchased from Guoyao Chemical Reagent Co. Compound c was obtained from Selleck Chemicals.

Cell culture and treatment. Human GEnCs were purchased from the ScienCell Research Laboratories, Inc. and cultured under standard cell culture conditions at $37^{\circ} \mathrm{C}$ with $5 \% \mathrm{CO}_{2}$ in a humidified incubator using endothelial cell medium (ECM; Shanghai Zhong Qiao Xin Zhou Biotechnology Co., Ltd.), supplemented with $100 \mathrm{U} / \mathrm{ml}$ penicillin, $100 \mu \mathrm{g} / \mathrm{ml}$ streptomycin, $10 \%$ (v/v) FBS and 1\% endothelial cell growth factor (Zhongqiaoxinzhou Biotech). Cells between passages 3 and 8 were used for the experiments. To study the role of AMPK in the protective effect of PF on HG-induced-GEnCs injury, GEnCs were preincubated with $20 \mu \mathrm{M}$ Compound $\mathrm{c}$ for $1 \mathrm{~h}$ and subsequently treated with $\mathrm{HG}(30 \mathrm{mM})$ in the presence or absence of PF for $48 \mathrm{~h}$. Next, the permeability of GEnCs to FITC-dextran was determined.

Endothelial permeability assays. FITC-dextran was used to measure permeability of GEnC monolayers. GEnC monolayers were cultured on the apical chamber of the Transwell inserts $(0.4 \mu \mathrm{m}$ pore size; Corning, Inc.) until they reached $100 \%$ confluence and were subsequently treated with $5.5 \mathrm{mM}$ glucose (control), $30 \mathrm{mM} \mathrm{HG}$ or mannitol (5.5 mM glucose $+24.5 \mathrm{mM}$ mannitol) with or without PF $(25-200 \mu \mathrm{M})$ for the indicated time periods. Dextran was dissolved in ECM and added to the apical chamber at a concentration of $2.0 \mathrm{~g} / 1$ following different experimental treatments. Following $1 \mathrm{~h}$ of incubation, the amount of fluorescence of FITC-dextran in the basolateral chamber was measured using fluorescence spectrophotometry with an excitation and emission wavelength of 490 and $520 \mathrm{~nm}$, respectively. The results are presented as fold-change following normalization to the control group.

Small interfering RNA (siRNA)-mediated knockdown. Specific and negative control siRNAs were purchased from Guangzhou RiboBio Co., Ltd. siRNA-AMPK (40 nM; cat. no. siB1456165958-1-5) was transfected into GEnCs using Dharma FECT transfection reagent (Thermo Fisher Scientific, Inc.) according to the manufacturer's protocol. The negative control group were GEnCs transfected with scrambled RNA (40 nM, cat. no. siN0000001-1-5). Following transfection, the cells were incubated for $24 \mathrm{~h}$ and used for further experiments.

Reverse transcription-quantitative PCR (RT-qPCR). Total RNA was extracted from renal tissues using TRIzol ${ }^{\circledR}$ reagent and cDNA was synthesized using a PrimeScript reverse transcription reagent kit according to the manufacturer's protocol. RT-qPCR was performed using a LightCycler96 ${ }^{\circledR}$ (Roche Diagnostics GmbH) and SYBR-Green master mix. The RT-qPCR cycling conditions were as follows: Initial denaturation at $95^{\circ} \mathrm{C}$ for $30 \mathrm{sec}$ followed by 45 cycles of denaturation at $95^{\circ} \mathrm{C}$ for $5 \mathrm{sec}$ and annealing $60^{\circ} \mathrm{C}$ for $30 \mathrm{sec}$. The species-specific primer sequences were as follows: Mouse occludin-1 forward, 5'-CTACGGAGGTGGCTATGGAG-3' and reverse, 5'-AAGGAAGCGATGAAGCAGAA-3'; mouse ZO-1 forward, 5'-ATGACTCCTGACGGTTGGTC-3' and reverse, 5'-GGCTCCAACAAGGTAATTCG-3'; and mouse $\beta$-actin forward, 5'-ACTGCTCTGGCTCCTAGCAC-3' and reverse, 5'-ACATCTGCTGGAAGGTGGAC-3'. Gene expression was calculated using the $2^{-\Delta \Delta \mathrm{Cq}}$ method (19). The data are expressed as the fold-change normalized to $\beta$-actin.

Western blot analysis. Total protein was extracted from cells using RIPA lysis buffer and the protein concentration was determined using the BCA method according to the manufacturer's protocol. A total of $35 \mu \mathrm{g}$ protein/lane was loaded onto a SDS-PAGE (10\%) and separated at $120 \mathrm{~V}$. The proteins were transferred to PVDF membranes and blocked in 5\% non-fat milk for $1 \mathrm{~h}$ at room temperature. Subsequently, the blots were incubated with primary antibodies against ZO-1 (1:500), occludin-1 (1:500), AMPK (1:500), hpa-1 (1:500), p-AMPK $\alpha(\operatorname{Thr} 172,1: 300)$ and $\beta$-actin $(1: 500)$ at $4^{\circ} \mathrm{C}$ overnight. The blots were incubated with HRP-conjugated goat anti-mouse (Boster Biological Technology; cat. no. BA1050) or HRP-conjugated goat anti-rabbit (Boster Biological Technology; cat. no. BA1054) diluted with secondary antibody dilution buffer (Beyotime Institute of Biotechnology, cat. no. P0023D) for $1 \mathrm{~h}$ at room temperature. The bands were visualized using a BeyoECL Plus kit. Densitometry analysis was performed using ImageJ version $1.45 \mathrm{~s}$ (National Institutes of Health). The protein expression levels were normalized to those of $\beta$-actin.

Animal model. The animal experimental protocol was approved by the Ethics Committee of Animal Experiments of the Central South University and was performed in accordance with the Guidelines for the Care and Use of Laboratory 
A
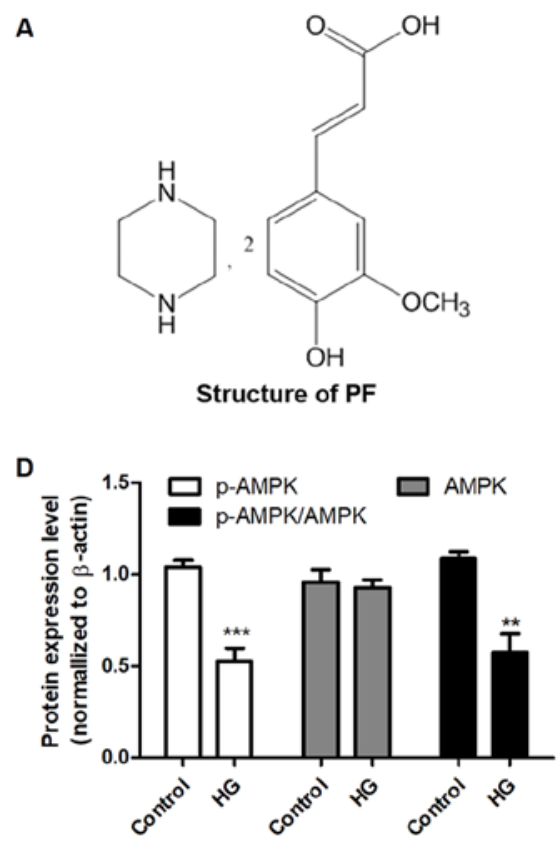

B

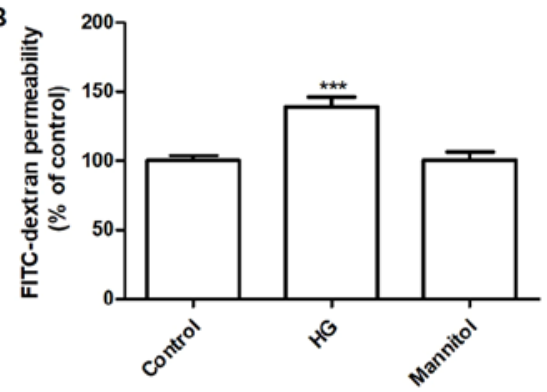

$E$

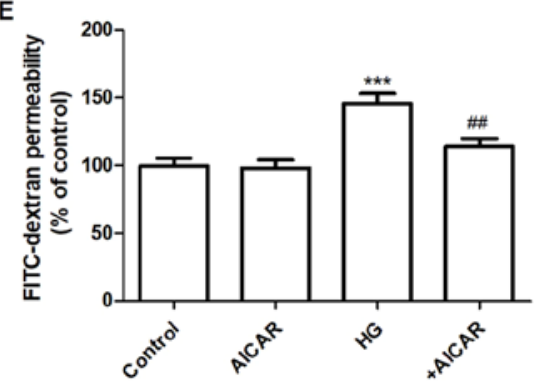

C

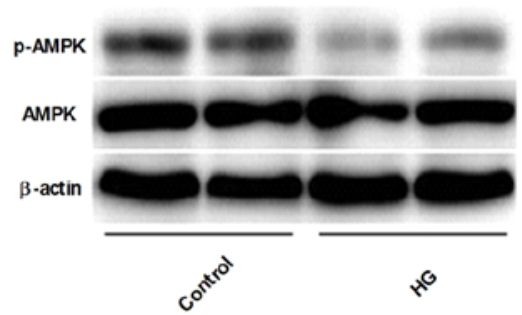

$\mathbf{F}$

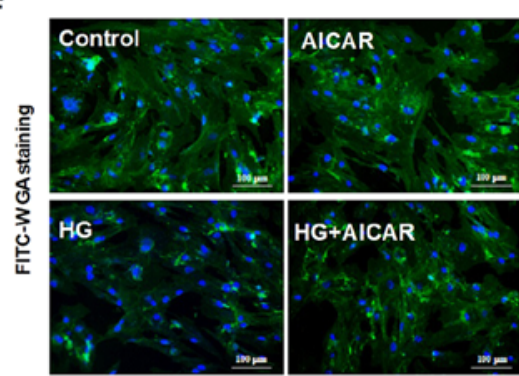

Figure 1. AMPK mediates HG-induced filtration barrier injury of GEnCs. (A) Structure of PF. (B) Effect of HG on the permeability of GEnCs to FITC-dextran. (C) Western blot analysis of p-AMPK $\alpha$ (Thr-172) and total AMPK. (D) Densitometric analysis of AMPK, p-AMPKa (Thr-172) and the p-AMPK $\alpha$ (Thr-172)/AMPK ratio. (E) Effect of AICAR (1 mM) on the HG-induced permeability of GEnCs. (F) Representative images of glycocalyx staining with FITC-WGA. Data are presented as the mean \pm standard deviation of three repeats. ${ }^{* *} \mathrm{P}<0.01,{ }^{* * * *} \mathrm{P}<0.001$ vs. control group; ${ }^{\# \#} \mathrm{P}<0.01$ vs. HG group. AMPK, adenosine monophosphate-activated protein kinase; HG, high glucose; GEnCs, glomerular endothelial cells; FITC, fluorescein isothiocyanate; p-, phosphorylated-; AICAR, 5-aminoimidizole-4-carboxamide riboside; WGA, wheat germ agglutinin.

Animals (20). Male C57BL/6J mice, aged 8 weeks (weight, 19-22 g), were purchased from the Experimental Animal Center of SiLaiKeJingDa. The animals were maintained under a constant $12 \mathrm{~h}$ light/dark cycle at $22 \pm 2^{\circ} \mathrm{C}$ and allowed free access to food and water at least for 1 week. Type 1 diabetic mice were established using a streptozotocin (STZ) injection as previously described (16). Briefly, the mice were intraperitoneally injected for 5 consecutive days with STZ $(65 \mathrm{mg} / \mathrm{kg}$ body weight). After 2 days, the levels of blood glucose were measured by the mouse tail snipping method using a glucometer (Sannuo Biotech Ltd.). Mice with blood glucose levels $\geq 16.7 \mathrm{mmol} / 1$ were considered diabetic. Normal control mice were injected with citrate buffer solution ( $0.1 \mathrm{M}, \mathrm{pH} 4.0)$. Then, 1 week after blood glucose stabilization, diabetic mice were divided into the two following groups ( $n=15$ per group): The diabetic mice group were treated with vehicle (DM) and the PF group of mice treated with PF (100 mg/kg, DM+PF). PF was provided by intragastric administration daily for 12 weeks. All mice were anaesthetized using sodium pentobarbital $(50 \mathrm{mg} / \mathrm{kg}$ body weight) and blood samples (1-1.2 ml) were collected from the retro-orbital sinus after eyeball removal. Finally, the mice were sacrificed by exsanguination under anesthesia $(50 \mathrm{mg} / \mathrm{kg}$ sodium pentobarbital) and the kidney tissues were collected. Collected blood was left to stand at room temperature for $1 \mathrm{~h}$, and then centrifuged at $2,000 \mathrm{x} \mathrm{g}$ at $4^{\circ} \mathrm{C}$ for $15 \mathrm{~min}$. The supernatant was transferred to a new tube and stored at $-80^{\circ} \mathrm{C}$.

Immunofluorescence analysis. Renal tissues were fixed in $4 \%$ paraformaldehyde at $4^{\circ} \mathrm{C}$ overnight, embedded in paraffin and cut into $5 \mu \mathrm{m}$-thick slices. The slices were processed by dewaxing, gradient alcohol dehydration, antigen repair with $10 \mathrm{mM}$ sodium citrate ( $\mathrm{pH} \mathrm{6.0,} \mathrm{microwaved} \mathrm{for} 10 \mathrm{~min}$ ) and finally washed in PBS three times. The sections were incubated with FITC-WGA $(10 \mu \mathrm{g} / \mathrm{ml})$ at room temperature for $2 \mathrm{~h}$, stained with DAPI at room temperature for $10 \mathrm{~min}$ and examined using fluorescence microscopy (Zeiss AG; magnification, $\mathrm{x} 400$ ). Immunofluorescence was performed by incubating the slides with ZO-1 (1:200)/CD31 (1:200) or Sdc-1 (1:200)/CD31 (1:200) overnight at $4^{\circ} \mathrm{C}$. The slides were incubated with the corresponding anti-FITC-conjugated secondary antibody (Beyotime Institute of Biotechnology; cat. no. A0562; 1:500) or Cy3-conjugated secondary antibody (Beyotime Institute of Biotechnology; cat. no. A0516; 1:500) diluted with secondary antibody dilution buffer for $1 \mathrm{~h}$ at room temperature in the dark. The cell nuclei were stained with DAPI at room temperature for $5 \mathrm{~min}$. Finally, the images were captured using fluorescence microscopy (magnification, x400).

Immunohistochemical analysis. The renal tissue slices were incubated overnight with anti-p-AMPK $\alpha$ (Thr-172) or Hpa-1 antibodies at $4^{\circ} \mathrm{C}$ overnight, followed by incubation with the corresponding secondary antibody conjugated with horseradish peroxidase (Boster Biological Technology; cat. no. BM3894; diluted with secondary antibody dilution buffer, 1:1,000) and 3,3'-diaminobenzidine peroxidase substrate. Finally, the sections were stained with hematoxylin at room temperature for $1 \mathrm{~min}$ and evaluated using conventional light microscopy (Olympus Corporation; magnification, x400).

Transmission electron microscopy. The kidney tissues were fixed in $2.5 \%$ glutaraldehyde at $4^{\circ} \mathrm{C}$ overnight and post-fixed in $1 \%$ osmium tetroxide at $4^{\circ} \mathrm{C}$ for $2 \mathrm{~h}$. Following dehydration, the samples were embedded in EMbed 812 resin (Thermo Fisher Scientific, Inc.) and polymerized at $60^{\circ} \mathrm{C}$ for $48 \mathrm{~h}$. The 
A

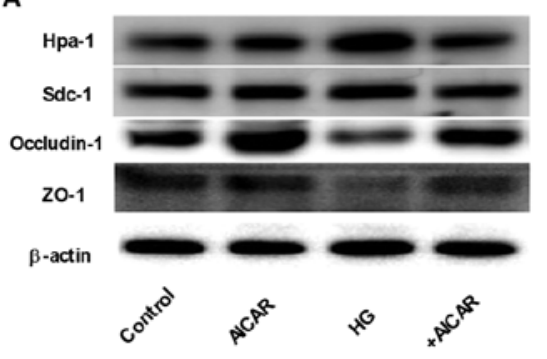

D

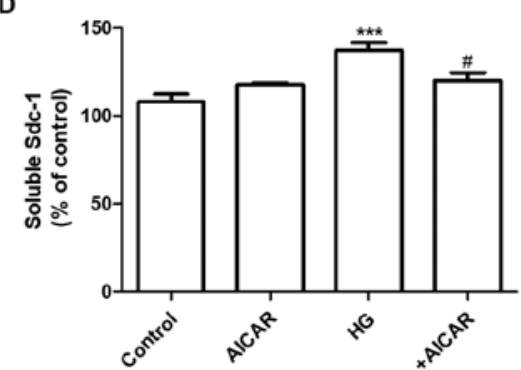

B

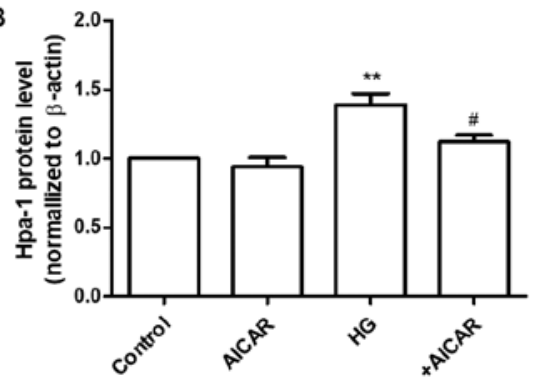

E

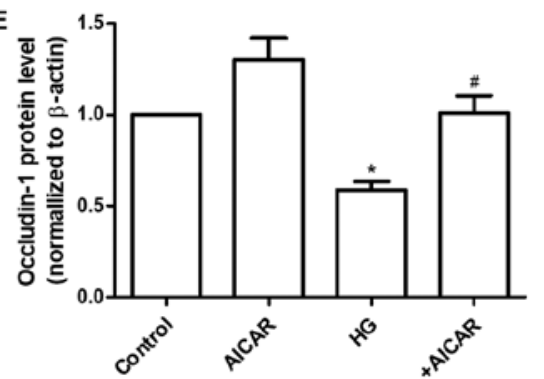

C

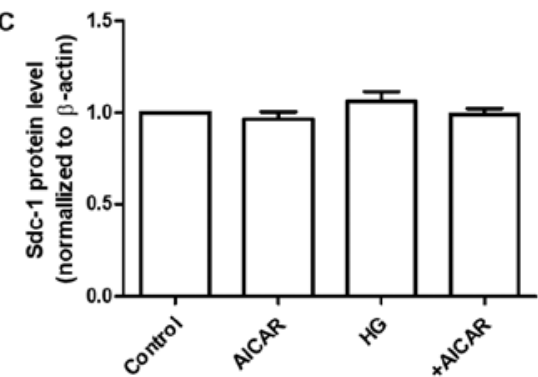

$\mathrm{F}$

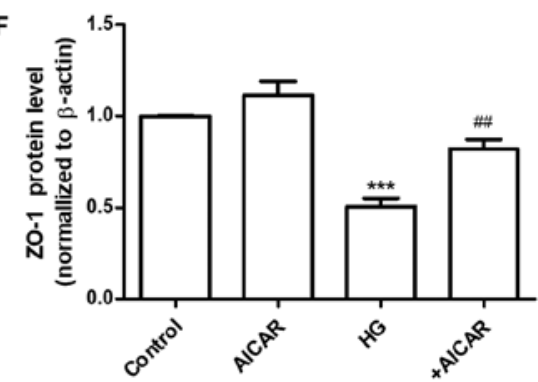

Figure 2. AMPK regulates the expression of Hpa-1 and TJ proteins. (A) Western blot analysis of Hpa-1, Sdc-1, occludin-1 and ZO-1 expression. Densitometric analysis of (B) Hpa-1 and (C) Sdc-1 expression. (D) Expression levels of Sdc-1 in the cell culture supernatant. (E) Densitometric analysis of occludin-1 and (F) ZO-1 expression. Data are presented as the mean \pm standard deviation of three repeats. ${ }^{*} \mathrm{P}<0.05,{ }^{* *} \mathrm{P}<0.01,{ }^{* * * *} \mathrm{P}<0.001$ vs. the control group; ${ }^{*} \mathrm{P}<0.05$, ${ }^{* \#} \mathrm{P}<0.01$ vs. HG. AMPK, adenosine monophosphate-activated protein kinase; Hpa-1, heparanase-1; TJ, tight junction; Sdc-1, syndecan-1; ZO-1, Zonula occludens-1; HG, high glucose.

ultrathin sections $(60-80 \mathrm{~nm})$ were cut and stained with $1 \%$ uranyl acetate at room temperature for $10 \mathrm{~min}$, followed by $2 \%$ lead citrate buffer for $2 \mathrm{~min}$ at $37^{\circ} \mathrm{C}$. The structure of the glomerulus was observed using a Hitachi High-Tech 7700 electron microscope (magnification, x5,000).

Determination of the expression levels of soluble Sdc-1. The levels of soluble Sdc-1 in the cell culture supernatant or serum were measured using the Sdc-1 ELISA kits according to the manufacturer's protocols.

Determination of glycocalyx via endothelial surface analysis. Following treatment, GEnCs were washed with PBS and fixed in 4\% paraformaldehyde at room temperature for $10 \mathrm{~min}$. Subsequently, the cells were incubated with FITC-WGA $(10 \mu \mathrm{g} / \mathrm{ml})$ at room temperature for $30 \mathrm{~min}$. Finally, the images were captured using fluorescence microscopy (magnification, $\mathrm{x} 400$ ).

Statistical analysis. Data are presented as the mean \pm standard error of at least three independent experiments. Data were analyzed using SPSS software (version 17.0; SPSS, Inc.). A one-way ANOVA followed by a post-hoc Tukey's test was performed to compare difference between multiple groups. $\mathrm{P}<0.05$ was considered to indicate a statistically significant difference.

\section{Results}

AMPK mediates $H G$-induced filtration barrier injury of GEnCs. The effects of HG on the permeability of the GEnC barrier to FITC-dextran were examined. HG (30 mM) increased the permeability of FITC-dextran after $48 \mathrm{~h}$, whereas mannitol did not have an effect for $48 \mathrm{~h}$ (Fig. 1B). Subsequently, the effects of $\mathrm{HG}$ on the expression levels of
AMPK and p-AMPK $\alpha$ (Thr-172) were measured by western blot analysis. GEnCs treated with $\mathrm{HG}$ exhibited decreased levels of p-AMPK $\alpha$ (Thr-172), although the total expression levels of AMPK were not altered notably (Fig. 1C and D). The effects of AMPK on the permeability of GEnC to FITC-dextran were further examined using AICAR, a selective agonist of AMPK. As shown in Fig. 1E, GEnCs pretreated with AICAR $(1 \mathrm{mM})$ decreased the HG-induced permeability of GEnC to FITC-dextran. Similarly, the results of FITC-WGA staining demonstrated that AICAR significantly inhibited the decrease of the glycocalyx content in GEnC induced by HG (Fig. 1F). These data suggested that AMPK mediated HG-induced filtration barrier injury of GEnCs.

AMPK regulates the expression of $\mathrm{Hpa}-1$ and tight junction (TJ) proteins. Sdc-1 is the main constituent of the glycocalyx. This protein can be cleaved by Hpa-1, an enzyme capable of degrading polymeric heparan sulfate molecules of Sdc-1 into shorter chain length oligosaccharides (21). HG increased the expression levels of Hpa-1 without affecting the expression levels of Sdc-1 in GEnCs (Fig. 2A). Therefore, the expression levels of Sdc-1 in the cell culture supernatant were determined. The results demonstrated that the expression levels of Sdc-1 were increased in the HG group. These changes were significantly inhibited by co-incubation of the cells with AICAR (Fig. 2A-D). Furthermore, the effects of AICAR on the expression of TJ proteins, ZO-1 and occludin-1 were investigated. AICAR significantly inhibited the HG-induced downregulation of the expression of occludin-1 and ZO-1 at the protein level (Fig. 2A, E and F). These data suggested that AMPK mediated the HG-induced filtration barrier injury of GEnCs by regulation of the expression levels of Hpa-1 and TJ proteins.

PF attenuates $H G$-induced filtration barrier damage. The effects of PF on the permeability defects of GEnCs 
A
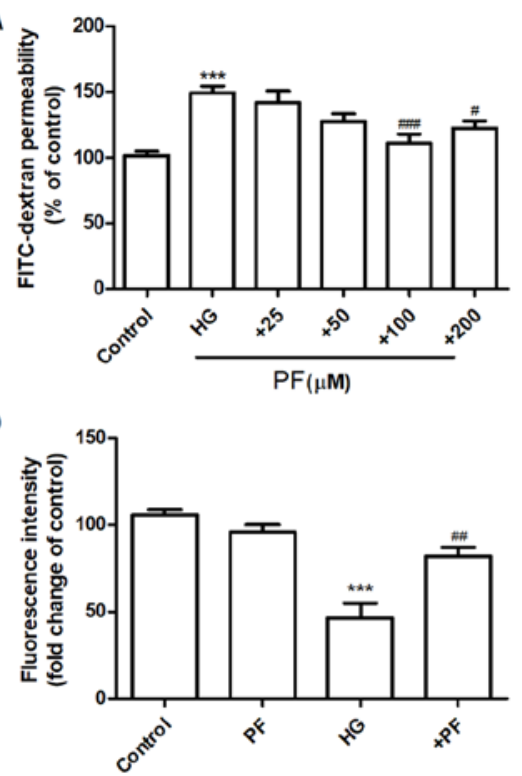

G

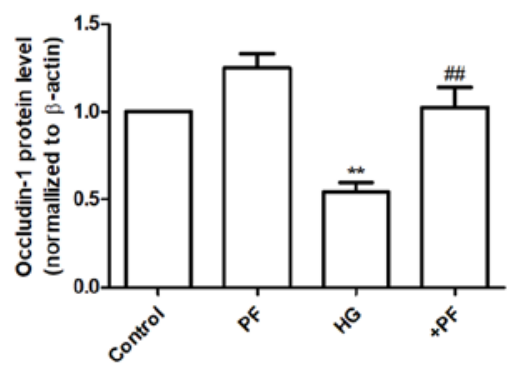

B

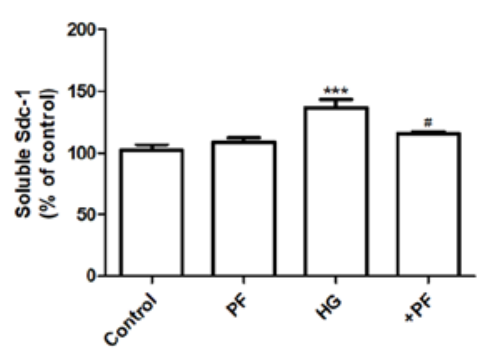

E

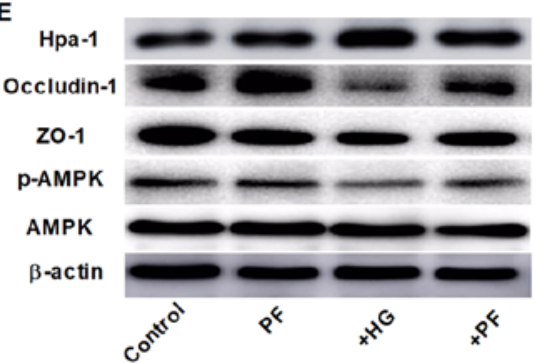

H

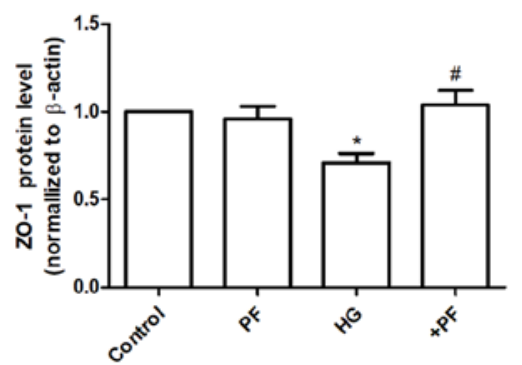

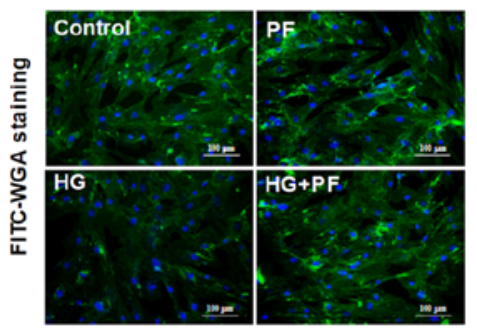

F

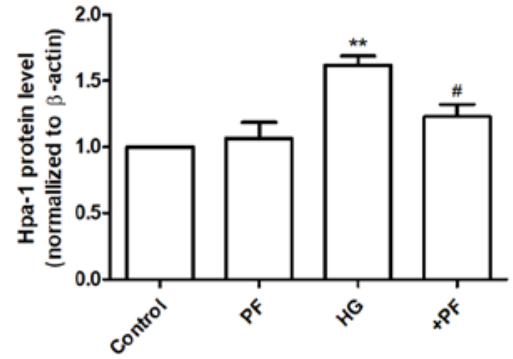

1

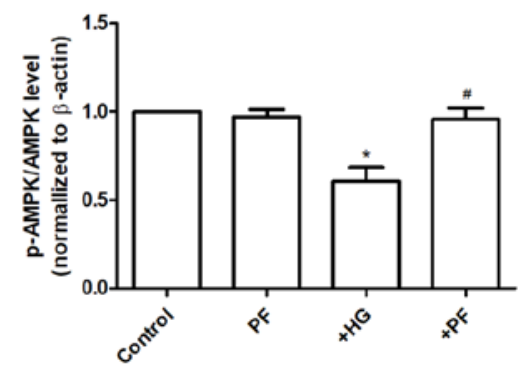

Figure 3. PF attenuates HG-induced filtration barrier damage. (A) Effects of various concentrations of PF on HG-induced permeability of GEnCs to FITC-dextran. (B) Expression levels of Sdc-1 in the cell culture supernatant. (C) Representative images of glycocalyx stained with FITC-WGA. (D) Quantification analysis of FITC-WGA staining. (E) Western blot analysis of Hpa-1, occludin-1, ZO-1, p-AMPK and total AMPK. Densitometry analysis of (F) Hpa-1, (G) occludin-1, (H) ZO-1 and (I) the p-AMPK/AMPK ratio. Data are presented as the mean \pm standard deviation of three repeats. ${ }^{*} \mathrm{P}<0.05,{ }^{* *} \mathrm{P}<0.01,{ }^{* * *} \mathrm{P}<0.001 \mathrm{vs}$. control group; ${ }^{\#} \mathrm{P}<0.05,{ }^{\# \#} \mathrm{P}<0.01,{ }^{\# \# \#} \mathrm{P}<0.001$ vs. HG group. PF, piperazine ferulate; HG, high glucose; GEnCs, glomerular endothelial cells; FITC, fluorescein isothiocyanate; WGA, wheat germ agglutinin; Hpa-1, heparanase-1; ZO-1, Zonula occludens-1; p-, phosphorylated-; AMPK, adenosine monophosphate-activated protein kinase.

induced by $\mathrm{HG}$ were assessed. Incubation of GEnCs with PF (100 and $200 \mu \mathrm{M})$ significantly inhibited the increase noted in the HG-induced permeability of GEnCs to FITC-dextran (Fig. 3A). The specific dose of PF used for further investigations was $100 \mu \mathrm{M}$. Preincubation of GEnCs with PF attenuated the increase of soluble Sdc-1 expression levels in the cell culture supernatant (Fig. 3B). Moreover, it increased the glycoclayx content of GEnCs (Fig. 3C and D). PF ameliorated the HG-induced increase in the expression levels of Hpa-1 protein in GEnCs (Fig. 3E and F). Similarly, PF inhibited the decrease in the expression levels of ZO-1 and occludin-1 in GEnCs exposed to HG (Fig. 3E, G and H). GEnCs incubated with PF exhibited a significant increase in the p-AMPK/AMPK ratio compared with those noted in the HG group (Fig. 3E and I). These finding suggested that PF inhibited HG-induced filtration barrier injury in GEnCs.

PF alleviates $H G$-induced filtration barrier injury via AMPK. The regulatory mechanism of $\mathrm{PF}$ on $\mathrm{HG}$-induced filtration barrier injury was further investigated. GEnCs were preincubated with Compound c (AMPK inhibitor, $20 \mu \mathrm{M}$ ) for $1 \mathrm{~h}$ and subsequently treated with $\mathrm{HG}(30 \mathrm{mM})$ in the presence or absence of $\mathrm{PF}$ for $48 \mathrm{~h}$. Compound $\mathrm{c}$ abolished the protective effects of $\mathrm{PF}$ on the HG-induced increased of the permeability of GEnCs to
FITC-dextran and increased the Sdc-1 levels in the cell culture supernatant (Fig. 4A and B). Following knockdown of AMPK in GEnCs by siRNA (Fig. S1), the effects of PF on the HG-induced upregulation of Hpa-1 expression and the downregulation of ZO-1 and occludin-1 were inhibited (Fig. 4C-F). Taken together, the data revealed that AMPK mediated the protective effects of PF on the HG-induced filtration barrier injury.

PF alleviates endothelial glycocalyx injury in vivo. In vitro experiments demonstrated that diabetic mice treated with PF for 12 weeks exhibited an increase in the number of GEnC fenestrations compared with that of the model group (Fig. 5A and B). Immunofluorescence analysis indicated that the glycocalyx content was increased in the glomerular cells of the PF-treated group compared with that of the diabetic mice (Fig. 5C and D). Immunohistochemical analysis indicated that PF significantly increased p-AMPK $\alpha$ (Thr-172) protein expression (Fig. 5E and F) and inhibited the increase in Hpa-1 protein levels in the glomerular cells of diabetic mice (Fig. 5G and H). Immunofluorescence analysis indicated that the expression levels of Sdc-1 (Fig. 6A) and ZO-1 (Fig. 6B) in GEnCs were increased in PF-treated mice compared with those noted in diabetic mice. In addition, treatment of diabetic mice with PF inhibited the increase noted in the expression levels of Sdc-1 in the serum 
A

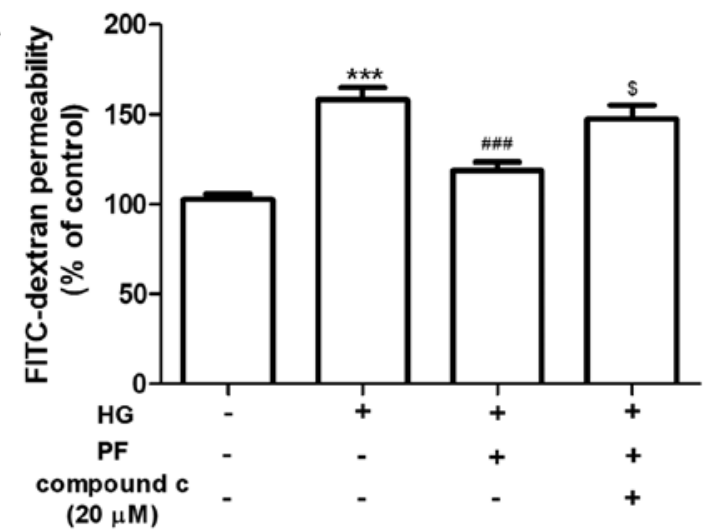

C

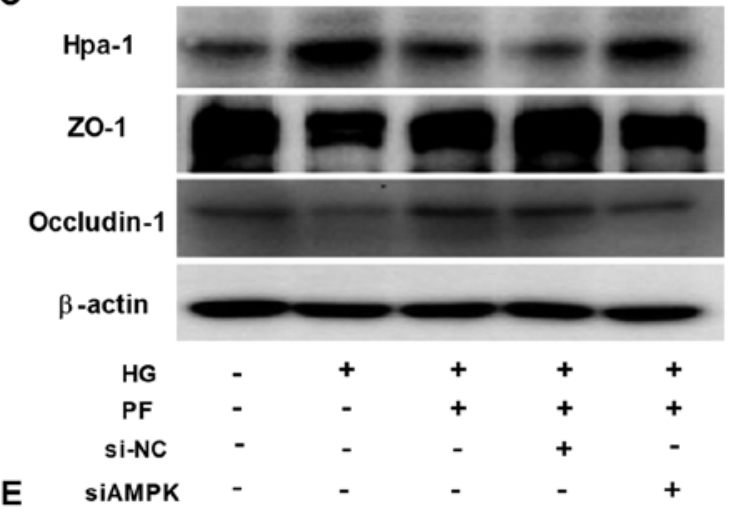

E

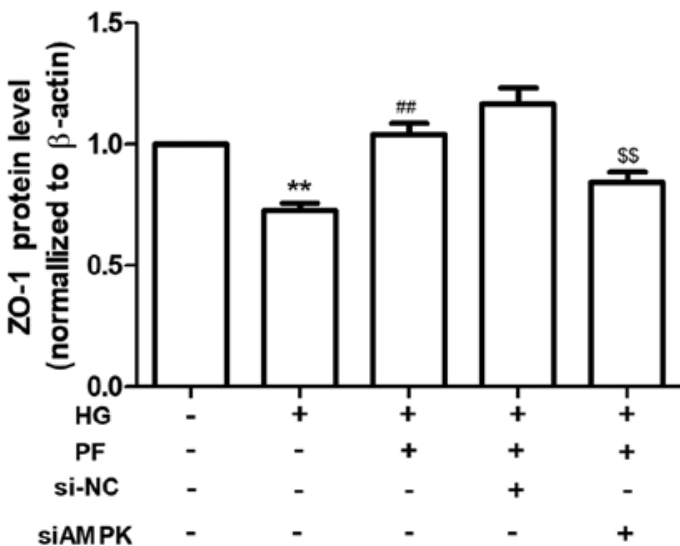

B

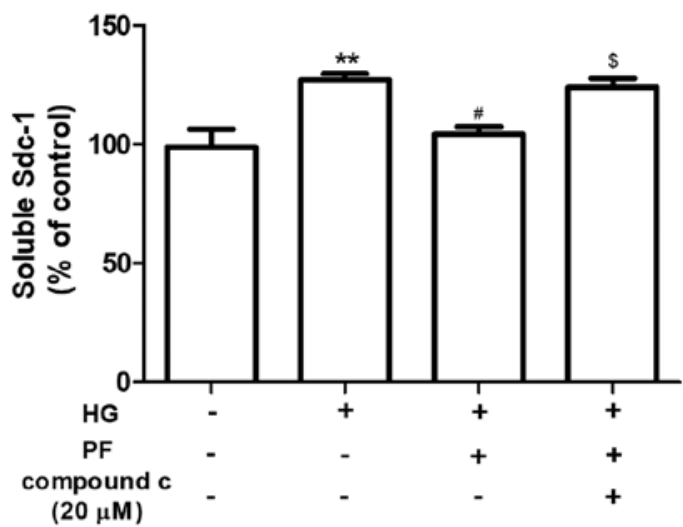

D
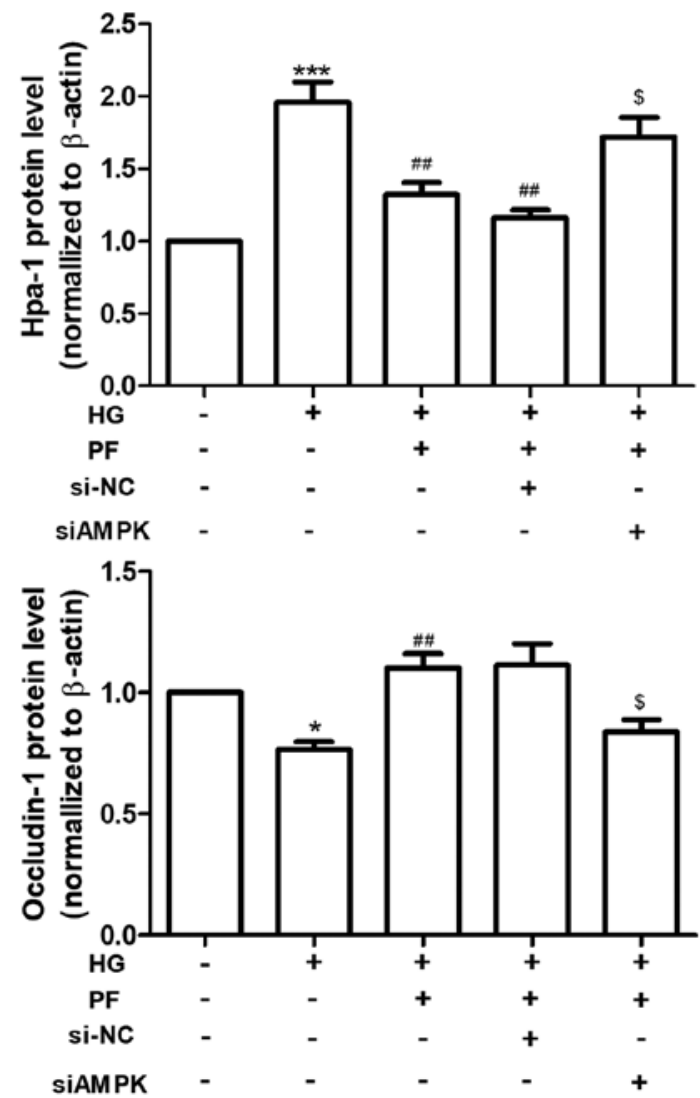

Figure 4. PF alleviates HG-induced filtration barrier injury via the AMPK pathway. (A) Permeability of GEnCs to FITC-dextran. (B) Expression levels of Sdc-1 in the cell culture supernatant. (C) Western blot analysis of Hpa-1, ZO-1 and occludin-1. Densitometry analysis of (D) Hpa-1, (E) ZO-1 and (F) occludin-1. Data are presented as the mean \pm standard deviation of three repeats. ${ }^{*} \mathrm{P}<0.05,{ }^{* *} \mathrm{P}<0.01,{ }^{* * * *} \mathrm{P}<0.001$ vs. control. ${ }^{\#} \mathrm{P}<0.05,{ }^{\# \#} \mathrm{P}<0.01,{ }^{\# \# "} \mathrm{P}<0.001$ vs. HG; ${ }^{\$} \mathrm{P}<0.05$, ${ }^{\$ \$} \mathrm{P}<0.01$ vs. HG + PF or siCon + HG + PF. PF, piperazine ferulate; HG, high glucose; AMPK, adenosine monophosphate-activated protein kinase; GEnCs, glomerular endothelial cells; FITC, fluorescein isothiocyanate; Sdc-1, syndecan-1; Hpa-1, heparanase-1; ZO-1, Zonula occludens-1; si, small interfering; NC, negative control.

(Fig. 6C). It concomitantly increased the expression levels of ZO-1 and occludin-1 in kidney tissues (Fig. 6D and E). Taken together, these finding indicated that $P F$ reduced GFB damage.

\section{Discussion}

Endothelial barrier injury is characterized by increased endothelial permeability and is associated with acute and chronic nephropathy (22). Emerging evidence has revealed that damage to the GEnC barrier plays an important role in the onset and progression of DN development (4). Our previous study demonstrated that PF attenuated the levels of $24 \mathrm{~h}$-albuminuria, blood urea nitrogen and serum creatinine in
STZ-induced DN mice (16), and it also reduced HG-induced mesangial cell injury (18). The results of the present study indicated that PF exerted a protective effect on GFB injury. The effects of PF were investigated on HG-induced filtration barrier injury of GEnCs, and the results suggested that this compound could reduce the damage to the glycocalyx degradation noted in GEnCs. These findings highlight a novel mechanism by which PF retards the progression of DN. This mechanism of action may restore endothelial filtration barrier injury.

Endothelial glycocalyx maintains the vascular function and plays an important role in vascular permeability, leucocyte, platelet adhesion as well as transduction of fluid shearing 
A

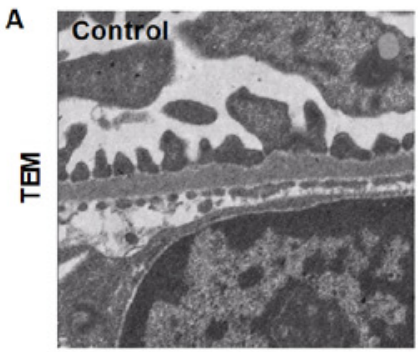

C

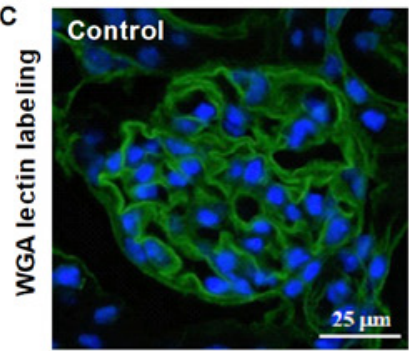

E

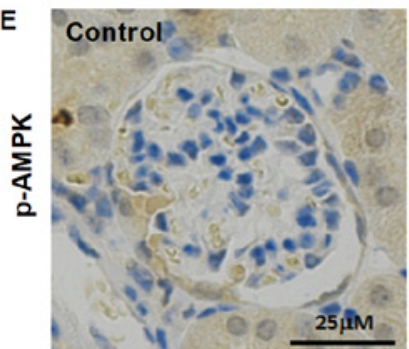

G

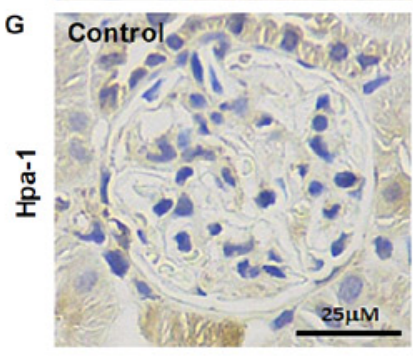

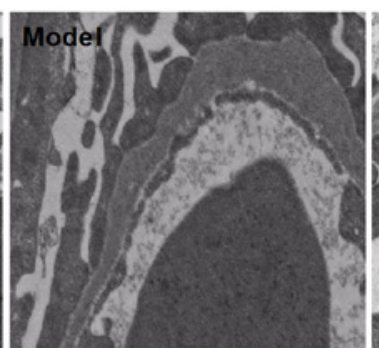
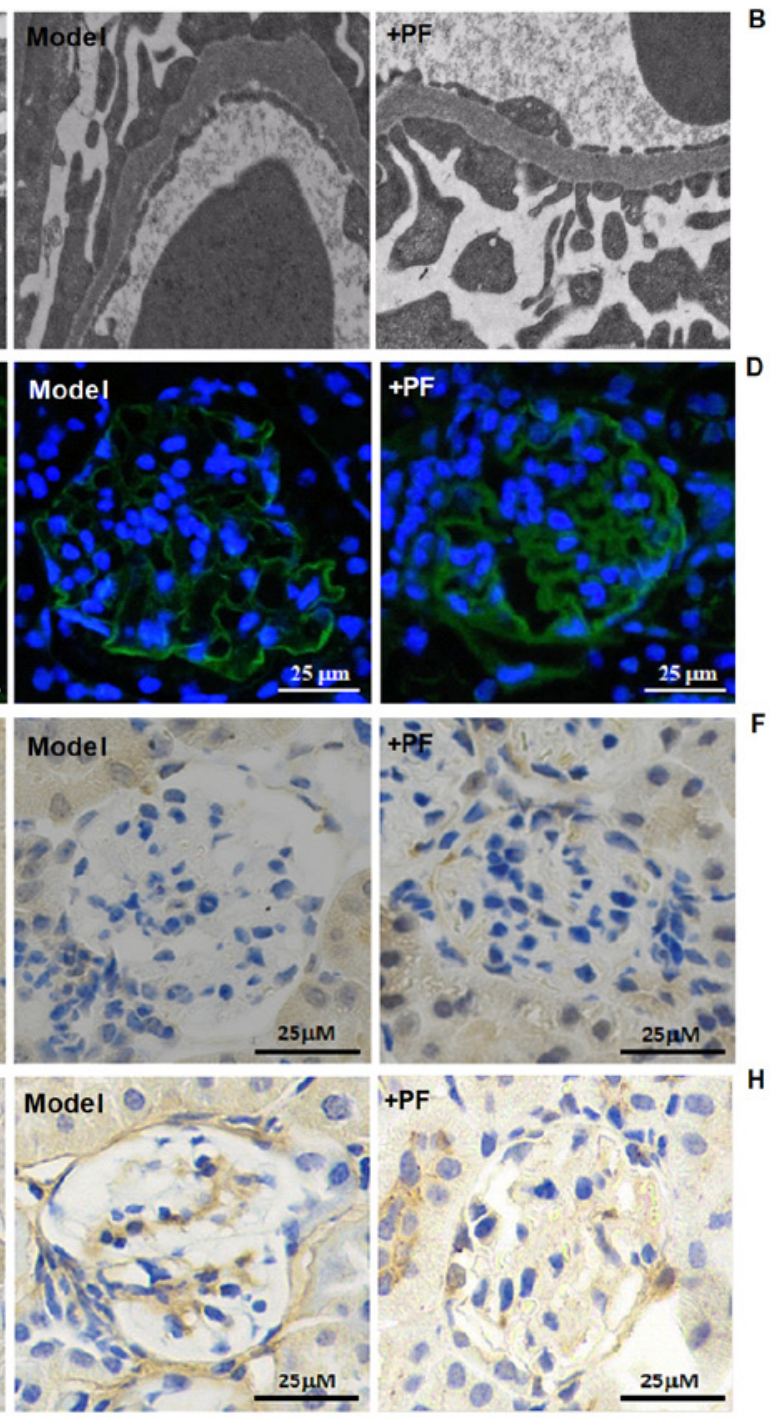
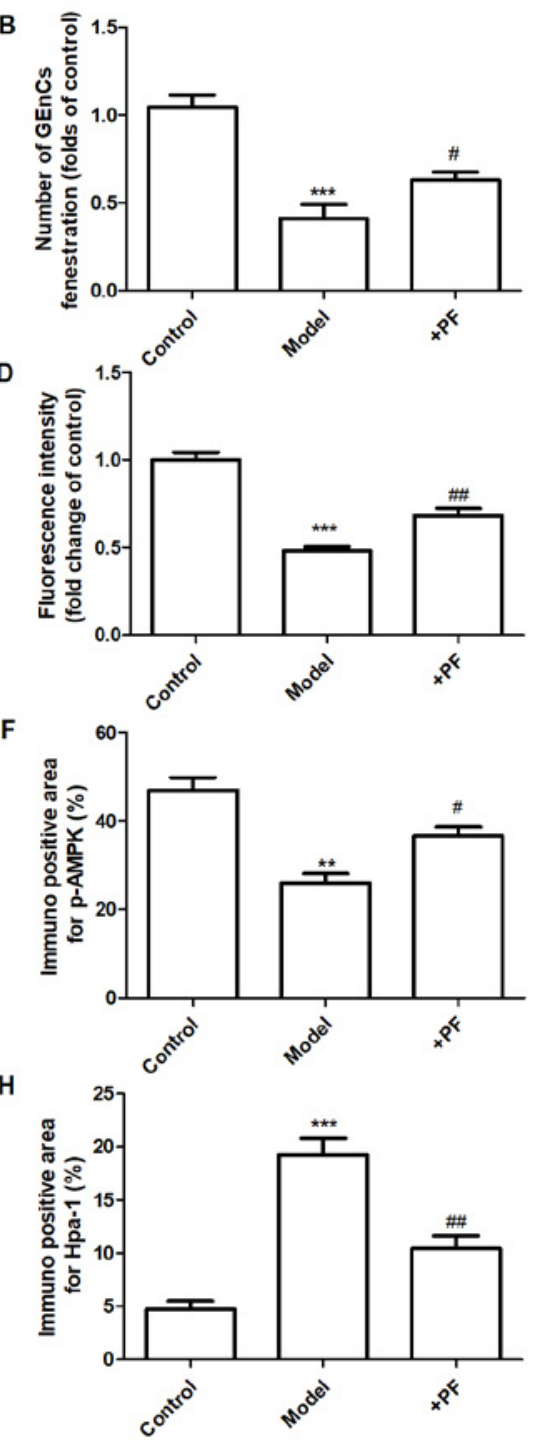

Figure 5. PF alleviates endothelial glycocalyx injury in vivo. (A) Transmission electron microscopy of glomeruli extracted from different groups of animals. (B) The number of glomerular endothelial fenestrations. (C) FITC-WGA staining for endothelial glycocalyx in the glomerulus. Magnification, $\mathrm{x} 400$. (D) Quantification analysis of FITC-WGA staining. (E) Immunohistochemical images of p-AMPK $\alpha$ (Thr-172). Magnification, $\mathrm{x} 400$. (F) Quantification analysis of immunohistochemical staining of p-AMPKa (Thr-172). (G) Immunohistochemical staining of Hpa-1. Magnification, x400. (H) Quantification analysis of immunohistochemical staining of Hpa-1. Data are presented as the mean \pm standard deviation of three repeats. ${ }^{* *} \mathrm{P}<0.01$, ${ }^{* * *} \mathrm{P}<0.01$ vs. the control group; ${ }^{\#} \mathrm{P}<0.05$, ${ }^{\# \#} \mathrm{P}<0.01$ vs. the model group. PF, piperazine ferulate; FITC, fluorescein isothiocyanate; WGA, wheat germ agglutinin; p-, phosphorylated-; AMPK, adenosine monophosphate-activated protein kinase; Hpa-1, heparanase-1.

forces $(23,24)$. FITC-WGA has been shown to bind to the glycoprotein of the glycocalyx (25). This marker was used to evaluate the effects of PF on glomerular endothelial glycocalyx. The data indicated that PF prevented HG-induced glycocalyx degradation in vivo and in vitro. Hpa-1 is an endo- $\beta$-D-glucuronidase that releases 5-7 $\mathrm{kDa}$ fragments of heparan sulfate from intact heparan sulfate chains of proteoglycans (26). In addition, Hpa-1 regulates the expression levels and turnover of syndecans (27). The results of the present study showed that HG increased the expression levels of Hpa-1 in vitro and in vivo. $\mathrm{PF}$ attenuated the increased expression of Hpa-1 in HG-induced GEnCs as well as in the glomeruli of diabetic mice. However, the expression levels of Sdc-1 did not decrease in HG-stimulated GEnCs. The effect of HG on the expression of Sdc-1 is similar to those of Singh et al (28). Immunofluorescence analysis indicated that Sdc-1 levels in the glomeruli of diabetic mice were decreased, whereas PF inhibited these changes. The possible reason for the discrepancy noted between the in vivo and in vitro experiments is the inconsistent exposure factors used for the different models. The degradation of glycocalyx in vivo may be affected by blood glucose levels, inflammatory factors and the course of diabetes (29). The exposure of GEnCs to HG involved a short term period $(24 \mathrm{~h})$, while the course of diabetes was completed following 12 weeks.

In the present study, the effects of PF on the TJ of GEnCs were also evaluated. The expression levels of ZO-1 were decreased in GEnCs of STZ-induced diabetic mice and in HG-treated GEnCs, respectively. PF treatment increased the expression levels of ZO-1. TJs between endothelial cells regulate the passage of ions and small molecules through the paracellular pathway and are involved in the pathogenesis of DN (30). The integrity of glomerular GFB is also maintained by $\mathrm{TJ}$ proteins that consist of at least 40 different proteins including transmembrane proteins, cytoplasm accessory $\mathrm{ZO}$ 


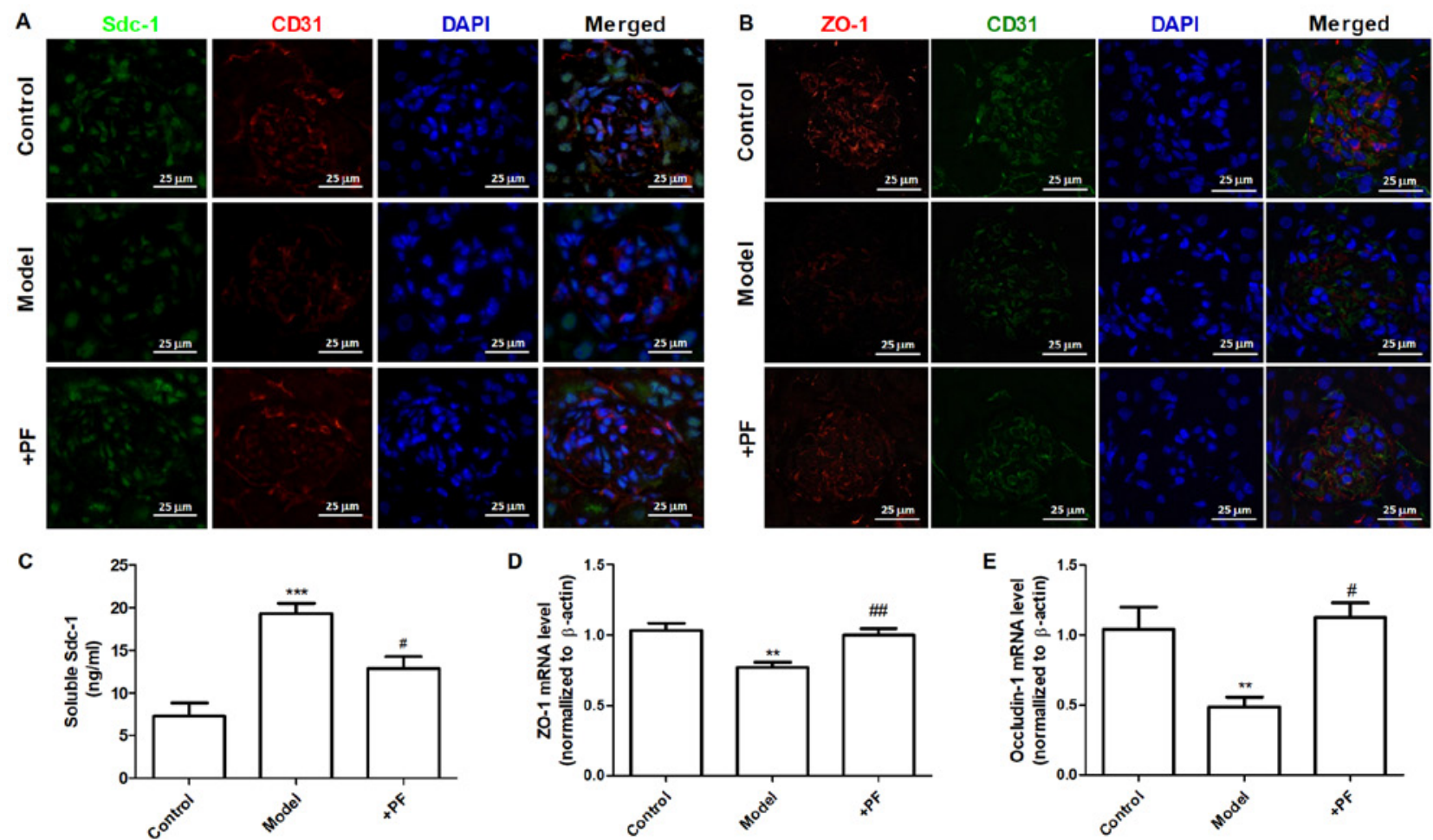

Figure 6. PF restores GFB injury. (A) Immunofluorescence images of Sdc-1 in the glomerulus. Magnification, x400. (B) Immunofluorescence image of ZO-1 in the glomerulus. Magnification, $\mathrm{x}$ 400. (C) Expression levels of Sdc-1 in the serum. (D) ZO-1 mRNA expression levels in the kidney tissues. (E) Occludin-1 mRNA expression levels in the kidney tissues. Data are presented as the mean \pm standard deviation of three repeats. ${ }^{* *} \mathrm{P}<0.01,{ }^{* * *} \mathrm{P}<0.001$ vs. the control group; ${ }^{\#} \mathrm{P}<0.05,{ }^{\# /} \mathrm{P}<0.01$ vs. model group. PF, piperazine ferulate; GFB, glomerular filtration barrier injury; Sdc-1, syndecan-1; ZO-1, Zonula occludens-1.

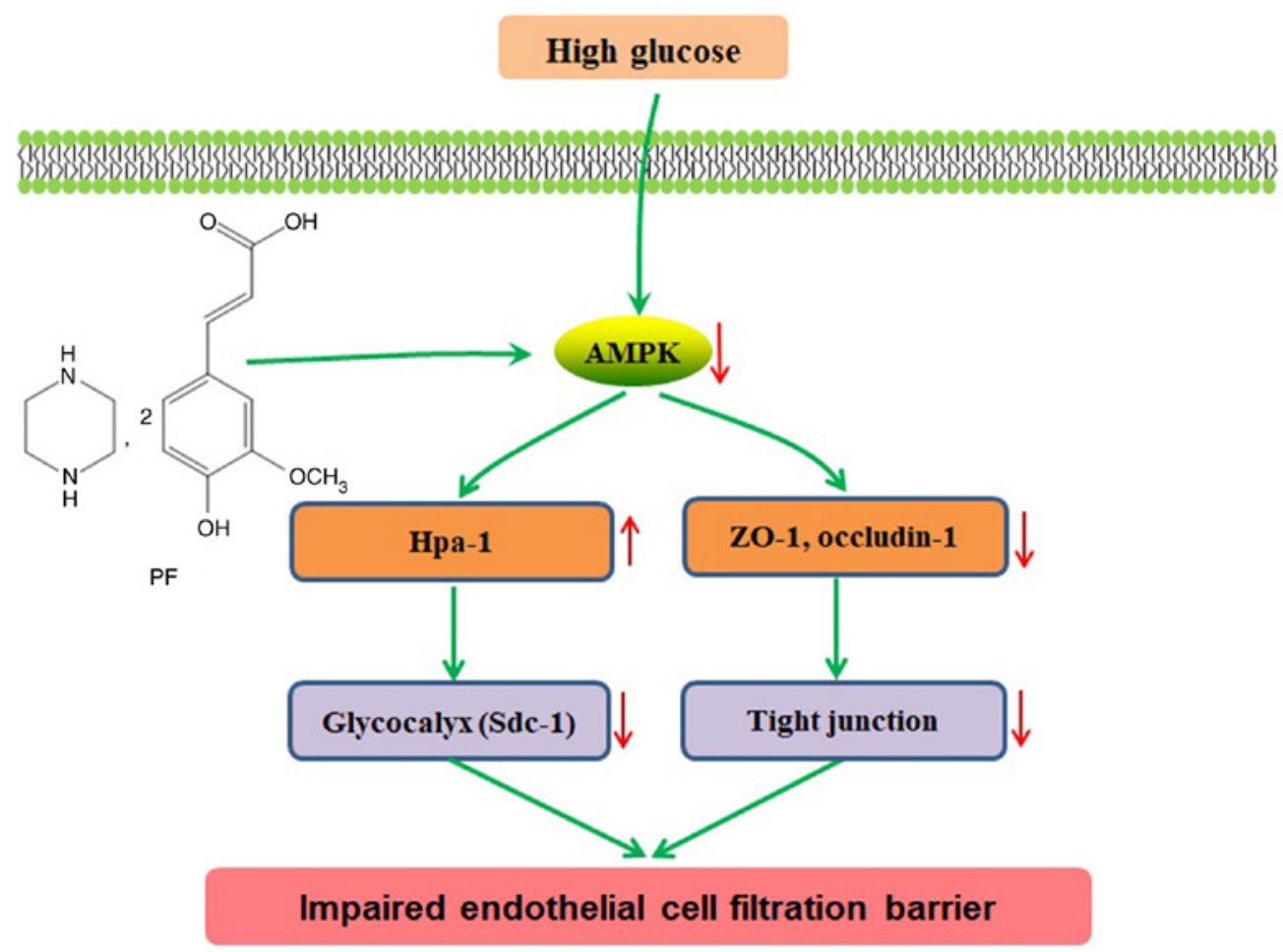

Figure 7. Schematic of pathways involved in the protective effects of PF on filtration barrier injury of GEnCs in diabetic mice. HG induces glycocalyx degradation in GEnCs and TJ damage between GEnCs in diabetic mice. PF inhibits glycocalyx degradation by regulation of AMPK. PF, piperazine ferulate; GEnCs, glomerular endothelial cells; HG, high glucose; TJ, tight junction; AMPK, adenosine monophosphate-activated protein kinase.

proteins and cytoskeletal proteins (30). HG influences the expression levels and/or redistribution of ZO-1, which leads to
TJ destruction and filtration barrier injury $(31,32)$. Thus, cell junctions may serve as a therapeutic target of DN. 
AMPK is an important energy-sensing enzyme that controls cellular energy metabolism and is considered a potential pharmacological target for the treatment of DN $(33,34)$. Phosphorylation of the $\alpha$ subunit at Thr-172 is essential for AMPK activity (35). Pharmacological activation of AMPK by AICAR results in restoration of the permeability of podocytes to albumin (36). It also increases the epithelial TJ assembly following calcium switch (37). In addition, ampkinone is an AMPK activator, which can be used to attenuate endothelial glycocalyx impairment induced by low shear stress (13). The results of the present study demonstrated that AMPK mediated HG-induced glycocalyx impairment and TJ injury in GEnCs. Based on western blotting, p-AMPK expression was decreased under HG conditions; however AMPK expression was not altered; whereas, PF increased the phosphorylation of AMPK following HG treatment. It is worth noting that treatment of GEnCs with the AMPK siRNA or inhibitor (compound c) abolished the protective effects of PF on the HG-induced filtration barrier injury. Specific molecular mechanisms may be involved in the phosphorylation of AMPK regulated by PF. A previous report indicated that ferulic acid, which is one of the components of $\mathrm{PF}$, regulated the phosphorylation of liver kinase $\mathrm{B} 1$ and AMPK in $\mathrm{C} 2 \mathrm{C} 12$ myoblasts via activation of sirtuin 1 (Sirt1) (38). An additional study demonstrated that ferulic acid stimulated the Sirt1/p-AMPK pathway in human cervical carcinoma cells (39). Therefore, PF may regulate AMPK phosphorylation via the Sirt1/LKB1 pathway.

In conclusion, the present study indicated that PF improved filtration barrier injury of GEnCs in a model of early DN, and that this action was mediated by AMPK (Fig. 7). Therefore, restoration of GFB injury via modulation of AMPK activity may serve an alternative approach for future treatment of DN.

\section{Acknowledgements}

Not applicable.

\section{Funding}

This work was supported financially by the Hunan Provincial Natural Scientific Foundation (grant no. 2020JJ5841), Hunan Traditional Chinese Medicine Science and Technology Project (grant no. 2021061), National Science and Technology Major Project Grant (grant no. 2020ZX09201-28), and Open Sharing Fund for the Large-scale Instruments of Central South University (grant no. CSUZC202056).

\section{Availability of data and materials}

The datasets used and/or analyzed during the present study are available from the corresponding author on reasonable request.

\section{Authors' contributions}

LYY and DXX designed the study. YYY, ZC and XDY performed the experiments. RRD and LXS contributed to data analysis. YYY wrote and revised the manuscript. All authors have read and approved the final manuscript. YYY and LXS confirm the authenticity of all the raw data.

\section{Ethics approval and consent to participate}

The animal experimental protocol used in the present study was approved by the Ethics Committee of Animal Experiments of the Central South University and was performed in accordance with the Guidelines for the Care and Use of Laboratory Animals.

\section{Patient consent for publication}

Not applicable.

\section{Competing interests}

The authors declare that they have no competing interests.

\section{References}

1. Boels MGS, Koudijs A, Avramut MC, Sol WMPJ, Wang G, van Oeveren-Rietdijk AM, van Zonneveld AJ, de Boer HC, van der Vlag J, van Kooten C, et al: Systemic monocyte chemotactic protein-1 inhibition modifies renal macrophages and restores glomerular endothelial glycocalyx and barrier function in diabetic nephropathy. Am J Pathol 187: 2430-2440, 2017.

2. Song K, Fu J, Song J, Herzog BH, Bergstrom K, Kondo Y, McDaniel JM, McGee S, Silasi-Mansat R, Lupu F, et al: Loss of mucin-type $\mathrm{O}$-glycans impairs the integrity of the glomerular filtration barrier in the mouse kidney. J Biol Chem 292: 16491-16497, 2017

3. Qi H, Casalena G, Shi S, Yu L, Ebefors K, Sun Y, Zhang W, D'Agati V, Schlondorff D, Haraldsson B, et al: Glomerular endothelial mitochondrial dysfunction is essential and characteristic of diabetic kidney disease susceptibility. Diabetes 66: 763-778, 2017.

4. Kusano T, Takano H, Kang D, Nagahama K, Aoki M, Morita M, Kaneko T, Tsuruoka S and Shimizu A: Endothelial cell injury in acute and chronic glomerular lesions in patients with $\operatorname{IgA}$ nephropathy. Hum Pathol 49: 135-144, 2016.

5. Salmon AH and Satchell SC: Endothelial glycocalyx dysfunction in disease: Albuminuria and increased microvascular permeability. J Pathol 226: 562-574, 2012.

6. Lavoz C, Matus YS, Orejudo M, Carpio JD, Droguett A, Egido J, Mezzano S and Ruiz-Ortega M: Interleukin-17A blockade reduces albuminuria and kidney injury in an accelerated model of diabetic nephropathy. Kidney Int 95: 1418-1432, 2019.

7. Dial AG, Ng SY, Manta A and Ljubicic V: The role of AMPK in neuromuscular biology and disease. Trends Endocrinol Metab 29: 300-312, 2018.

8. Packer M: Interplay of adenosine monophosphate-activated protein kinase/sirtuin-1 activation and sodium influx inhibition mediates the renal benefits of sodium-glucose co-transporter-2 inhibitors in type 2 diabetes: A novel conceptual framework. Diabetes Obes Metab 22: 734-742, 2020.

9. Lee MJ, Feliers D, Mariappan MM, Sataranatarajan K, Mahimainathan L, Musi N, Foretz M, Viollet B, Weinberg JM, Choudhury GG and Kasinath BS: A role for AMP-activated protein kinase in diabetes-induced renal hypertrophy. Am J Physiol Renal Physiol 292: F617-F627, 2007.

10. Eid AA, Ford BM, Block K, Kasinath BS, Gorin Y, Ghosh-Choudhury G, Barnes JL and Abboud HE: AMP-activated protein kinase (AMPK) negatively regulates Nox4-dependent activation of p53 and epithelial cell apoptosis in diabetes. J Biol Chem 285: 37503-37512, 2010.

11. Eisenreich A and Leppert U: Update on the protective renal effects of metformin in diabetic nephropathy. Curr Med Chem 24: 3397-3412, 2017.

12. Alhaider AA, Korashy HM, Sayed-Ahmed MM, Mobark M, Kfoury $\mathrm{H}$ and Mansour MA: Metformin attenuates streptozotocin-induced diabetic nephropathy in rats through modulation of oxidative stress genes expression. Chem Biol Interact 192: 233-242, 2011

13. Zhang J, Kong X, Wang Z, Gao X, Ge Z, Gu Y, Ye P, Chao Y, Zhu L, Li X and Chen S: AMP-activated protein kinase regulates glycocalyx impairment and macrophage recruitment in response to low shear stress. FASEB J 33: 7202-7212, 2019. 
14. Jianzhi S, Qizeng W, Bin L, Wenhui L, Yunpeng C, Chenrong F, Lin $\mathrm{Z}$ and Huiting C: Piperazine ferulate exerts antihypertensive effect and improves endothelial function in vitro and in vivo via the activation of endothelial nitric oxide synthase. Cell Mol Biol (Noisy-le-grand) 65: 119-124, 2019.

15. Liu Z, Pan J, Sun C, Zhou J and Li NA: Clinical effects of perazine ferulate tablets combined with eucalyptol limonene pinene enteric soft capsules for treatment of children with $\operatorname{Ig} \mathrm{A}$ nephropathy. Exp Ther Med 12: 169-172, 2016.

16. Yang YY, Shi LX, Li JH, Yao LY and Xiang DX: Piperazine ferulate ameliorates the development of diabetic nephropathy by regulating endothelial nitric oxide synthase. Mol Med Rep 19: 2245-2253, 2019.

17. Li D, Li B, Peng LX, Liu R and Zeng N: Therapeutic efficacy of piperazine ferulate combined with irbesartan in diabetic nephropathy: A systematic review and meta-analysis. Clin Ther 42: 2196-2212, 2020.

18. Yang YY, Deng RR, Chen Z, Yao LY, Yang XD and Xiang DX: Piperazine ferulate attenuates high glucose-induced mesangial cell injury via the regulation of 66 $^{\text {shc }}$. Mol Med Rep 23: 374, 2021

19. Livak KJ and Schmittgen TD: Analysis of relative gene expression data using real-time quantitative PCR and the 2(-Delta Delta C(T)) method. Methods 25: 402-408, 2001.

20. Jones-Bolin S: Guidelines for the care and use of laboratory animals in biomedical research. Curr Protoc Pharmacol Appendix 4: Appendix4B, 2012.

21. Yu S, Lv H, Zhang H, Jiang Y, Hong Y, Xia R, Zhang Q, Ju W, Jiang L, Ou G, et al: Heparanase-1-induced shedding of heparan sulfate from syndecan-1 in hepatocarcinoma cell facilitates lymphatic endothelial cell proliferation via VEGF-C/ERK pathway. Biochem Biophys Res Commun 485: 432-439, 2017.

22. Zafrani $L$ and Ince C: Microcirculation in acute and chronic kidney diseases. Am J Kidney Dis 66: 1083-1094, 2015

23. Okada H, Yoshida S, Hara A, Ogura S and Tomita H: Vascular endothelial injury exacerbates coronavirus disease 2019: The role of endothelial glycocalyx protection. Microcirculation 28: e12654, 2021.

24. Zhu T, Wang H, Wang L, Zhong X, Huang W, Deng X, Guo H, Xiong J, Xu Y and Fan J: Ginsenoside Rg1 attenuates high glucose-induced endothelial barrier dysfunction in human umbilical vein endothelial cells by protecting the endothelial glycocalyx. Exp Ther Med 17: 3727-3733, 2019.

25. Fukui M, Yamada M, Akune Y, Shigeyasu C and Tsubota K: Fluorophotometric analysis of the ocular surface glycocalyx in soft contact lens wearers. Curr Eye Res 41: 9-14, 2016.

26. Yang Y, Macleod V, Miao HQ, Theus A,ZhanF, Shaughnessy JD Jr, Sawyer J, Li JP, Zcharia E, Vlodavsky I and Sanderson RD: Heparanase enhances syndecan-1 shedding: A novel mechanism for stimulation of tumor growth and metastasis. J Biol Chem 282 13326-13333, 2007.

27. Rangarajan S, Richter JR, Richter RP, Bandari SK, Tripathi K, Vlodavsky I and Sanderson RD: Heparanase-enhanced shedding of syndecan-1 and its role in driving disease pathogenesis and progression. J Histochem Cytochem 68: 823-840, 2020.
28. Singh A, Friden V, Dasgupta I, Foster RR, Welsh GI, Tooke JE, Haraldsson B, Mathieson PW and Satchell SC: High glucose causes dysfunction of the human glomerular endothelial glycocalyx. Am J Physiol Renal Physiol 300: F40-F48, 2011.

29. Reine TM, Lanzalaco F, Kristiansen O, Enget AR, Satchell S, Jenssen TG and Kolset SO: Matrix metalloproteinase-9 mediated shedding of syndecan-4 in glomerular endothelial cells. Microcirculation: Jan 31, 2019 (Epub ahead of print).

30. Eftekhari A, Vahed SZ, Kavetskyy T, Rameshrad M, Jafari S, Chodari L, Hosseiniyan SM, Derakhshankhah H, Ahmadian E and Ardalan M: Cell junction proteins: Crossing the glomerular filtration barrier in diabetic nephropathy. Int J Biol Macromol 148: 475-482, 2020.

31. Rincon-Choles H, Vasylyeva TL, Pergola PE, Bhandari B, Bhandari K, Zhang JH, Wang W, Gorin Y, Barnes JL and Abboud HE: ZO-1 expression and phosphorylation in diabetic nephropathy. Diabetes 55: 894-900, 2006.

32. Ha TS, Choi JY, Park HY and Lee JS: Ginseng total saponin improves podocyte hyperpermeability induced by high glucose and advanced glycosylation endproducts. J Korean Med Sci 26: 1316-1321, 2011.

33. Kim Y and Park CW: New therapeutic agents in diabetic nephropathy. Korean J Intern Med 32: 11-25, 2017.

34. Kim Y and Park CW: Adenosine monophosphate-activated protein kinase in diabetic nephropathy. Kidney Res Clin Pract 35: 69-77, 2016.

35. Morales-Alamo D and Calbet JA: AMPK signaling in skeletal muscle during exercise: Role of reactive oxygen and nitrogen species. Free Radic Biol Med 98: 68-77, 2016.

36. Sharma K, Ramachandrarao S, Qiu G, Usui HK, Zhu Y, Dunn SR, Ouedraogo R, Hough K, McCue P, Chan L, et al: Adiponectin regulates albuminuria and podocyte function in mice. J Clin Invest 118: 1645-1656, 2008.

37. Zheng B and Cantley LC: Regulation of epithelial tight junction assembly and disassembly by AMP-activated protein kinase. Proc Natl Acad Sci USA 104: 819-822, 2007.

38. Chen X, Guo Y, Jia G, Zhao H, Liu G and Huang Z: Ferulic acid regulates muscle fiber type formation through the Sirt1/AMPK signaling pathway. Food Funct 10: 259-265, 2019.

39. Giovannini L and Bianchi S: Role of nutraceutical SIRT1 modulators in AMPK and mTOR pathway: Evidence of a synergistic effect. Nutrition 34: 82-96, 2017.

This work is licensed under a Creative Commons Attribution-NonCommercial-NoDerivatives 4.0 International (CC BY-NC-ND 4.0) License. 